Proyecciones Journal of Mathematics

Vol. 34, No 2, pp. 147-159, June 2015.

Universidad Católica del Norte

Antofagasta - Chile

\title{
On the stability and boundedness of certain third order non-autonomous differential equations of retarded type
}

\author{
Cemil Tunç \\ Yüzüncü Yil University, Turkey \\ Received : October 2014. Accepted : April 2015
}

\begin{abstract}
In this paper, based on the Lyapunov-Krasovskii functional approach, we obtain sufficient conditions which guarantee stability, uniformly stability, boundedness and uniformly boundedness of solutions of certain third order non- autonomous differential equations of retarded type. Our results complement and improve some recent ones.
\end{abstract}

Subjclass : [2010]34K20.

Keywords : Boundedness, stability, non-autonomous, retarded. 


\section{Introduction}

In this paper, we are interested in obtaining sufficient conditions for all solutions of the third order non-autonomous differential equation of retarded type

$x^{\prime \prime \prime}(t)=a(t) \varphi\left(x^{\prime \prime}(t-\tau)\right) x^{\prime \prime}(t)+b(t) \psi\left(x^{\prime}(t)\right)+h(x(t))+f\left(x(t), x^{\prime}(t-\tau)\right)+p(t)$, (1.1)

to be bounded and uniformly bounded, and in case $p(t) \equiv 0$, sufficient conditions for the zero solution to be stable and uniformly stable. Our motivation comes partially from a recent paper of El-Nahhas [11] who studied the stability of the autonomous differential equation of retarded type

$$
x^{\prime \prime \prime}(t)=a x^{\prime \prime}(t)+b x^{\prime}(t)+c x(t)+f\left(x(t), x^{\prime}(t-\tau)\right),
$$

where $a, b$ and $c$ are negative constants; $\tau(>0)$ is constant retardation; $f(0,0)=0$.

Differential equations of the type (1.1) and (1.2) have been shown to be useful in modeling many phenomena in various fields of science and engineering and in more recent years to problems in biomathematics (see, for example, Cronin-Scanlon [8] and Smith [19]). One special case of nonlinear differential equations of third order is what is known as the jerky dynamics equation

$$
x^{\prime \prime \prime}(t)+k_{1}\left(x(t), x^{\prime}(t)\right) x^{\prime \prime}(t)+k_{2}\left(x(t), x^{\prime}(t), x^{\prime \prime}(t)\right)=0
$$

that has gained some attention in the literature (see, Chlouverakis and Sprott [7], Eichhorn et al. [9], Elhadj and Sprott [10] and Linz [14]). Besides, qualitative properties of solutions of third order differential equations such as stability, instability, boundedness, oscillation, and periodicity of solutions have been studied by many authors; in this regard, we refer the reader to the monograph by Reissig et al. [17], and the recent papers of Adams et al. [1], Ademola and Arawomo [2], Afuwape and Adesina [3], Bai and Guo [5], Ogundare and Okecha [15], Rauch [16], Sadek [18], Tunç ([20][27]), Zhang and $\mathrm{Yu}[29]$ and the references cited therein. However, to the best of our knowledge, there exist few results on the mentioned qualitative behaviors of solutions for the non-autonomous third order differential equations of retarded type (see, the references of this paper). Motivated by the above discussions, the main purpose of this paper is to give some sufficient conditions for the stability, uniformly stability, boundedness and uniformly 
boundedness of solutions of equation (1.1). Our results complement and improve some recent ones.

One tool to be used here is the stability and boundedness theorems.

Let us consider non-autonomous delay differential equation

$$
x^{\prime}=F\left(t, x_{t}\right), x_{t}=x(t+\theta), \quad-r \leq \theta \leq 0, \quad t \geq 0,
$$

where $F: \Re_{+} \times C_{H} \rightarrow \Re^{n}, \Re_{+}=[0, \infty)$, is a continuous mapping, $F(t, 0)=$ 0 , and we suppose that $F$ takes closed bounded sets into bounded sets of $\Re^{n}$. Here $(C,\|\|$.$) is the Banach space of continuous function \phi:[-r, 0] \rightarrow$ $\Re^{n}$ with supremum norm; $C_{H}$ is the open $H$-ball in $C ; C_{H}:=\{\phi \in$ $\left.\left(C[-r, 0], \Re^{n}\right):\|\phi\|<H\right\}$. Let $S$ be the set of $\phi \in C$ such that $\|\phi\| \geq H$ denote by $S^{\bullet}$ the set of all functions $\phi \in C$ such that $|\phi(0)| \geq H$, where $H$ is large enough.

First, we will give some basic definitions.

Definition 1.1. (Burton [6]) Let $F(t, 0)=0$. The zero solution of equation (1.3) is stable if for each

$\varepsilon>0$ there is a $\delta>0$ such that $\left[t \geq 0, \quad\|\phi\|<\delta, \quad t \geq t_{0}\right]$ implies that $\left|x\left(t, t_{0}, \phi\right)\right|<\varepsilon$.

Definition 1.2. (Burton [6]) A continuous functions $W: \Re_{+} \rightarrow \Re_{+}$with $W(0)=0, W(s)>0$ if $s>0$, and $W$ strictly increasing is a wedge. (We denote wedges by $W$ or $W_{i}$, where $i$ an integer.)

Definition 1.3. (Burton [6]) Let $D$ be an open set in $\Re^{n}$ with $0 \in D$. A function $V: \Re_{+} \times D \rightarrow \Re_{+}$is called

(i) positive definite if $V(t, 0)=0$ and if there is a wedge $W_{1}$ with $V(t, x) \geq$ $W_{1}(|x|)$;

(ii) decresent if there is a wedge $W_{2}$ with $V(t, x) \leq W_{2}(|x|)$.

The following theorems are basic tools for our results. 
Theorem 1.1. (Burton [6]) Let $V: \Re_{+} \times C_{H} \rightarrow \Re_{+}$be continuous, (where $V$ is Lyapunov functional for equation (1.3). If

(i) If $W_{1}(|\phi(0)|) \leq V(t, \phi), V(t, 0)=0$, and

$V^{\prime}\left(t, x_{t}\right) \leq 0$, then the zero solution of equation (1.3) is stable;

(ii) If $W_{1}(|\phi(0)|) \leq V(t, \phi) \leq W_{2}(\|\phi\|), V(t, 0)=0$, and

$V^{\prime}\left(t, x_{t}\right) \leq 0$, (where $W_{1}$ and $W_{2}$ are wedges), then the zero solution of equation (1.3) is uniformly stable.

Theorem 1.2. (Yoshizawa [28]) Suppose that there exists a continuous Lyapunov functional $V(t, \phi)$ defined for all $t \in \Re_{+}$and $\phi \in S^{\bullet}$, which satisfies the following conditions;

(i) $\mathrm{a}(|\phi(0)|) \leq V(t, \phi) \leq b_{1}(|\phi(0)|)+b_{2}(\|\phi\|)$,

where $a(r), b_{1}(r), b_{2}(r) \in C I,(C I$ denotes the families of continuous increasing functions), and are positive for $r>H$ and $a(r)-b_{2}(r) \rightarrow \infty$ as $r \rightarrow \infty$;

(ii) $V^{\prime}(t, \phi) \leq 0$.

Then, the solutions of equation (1.3) are uniformly bounded.

\section{Main results}

We consider the nonlinear third order differential equation with constant delay $\tau$,

$x^{\prime \prime \prime}(t)=a(t) \varphi\left(x^{\prime \prime}(t-\tau)\right) x^{\prime \prime}(t)+b(t) \psi\left(x^{\prime}(t)\right)+h(x(t))+f\left(x(t), x^{\prime}(t-\tau)\right)+p(t)$,

where $\Re=(-\infty, \infty), \Re_{+}=[0, \infty), a(t)$ and $b(t)$ are negative and continuous functions in $\Re_{+}=[0, \infty), \varphi: \Re \rightarrow \Re, \psi: \Re \rightarrow \Re, h: \Re \rightarrow \Re$, $f: \Re^{2} \rightarrow \Re$ and $p: \Re_{+} \rightarrow \Re$ are continuous with $\psi(0)=0, h(0)=0$, $f(0,0)=0$, and $\tau$ is a positive constant.The continuity of the functions $a(t), b(t), \varphi, \psi, h, f$ and $p$ guarantees the existence of the solutions, and we assume that $\varphi, \psi, h$ and $f$ satisfy local Lipschitz conditions so that we have uniqueness of solutions to initial value problems as well (see, Èl'sgol'ts [12]), and the functions $h, f$ and $b(t)$ are differentiable.

We can write equation (2.1) as the system

$$
\begin{aligned}
& x^{\prime}(t)=y(t), \\
& y^{\prime}(t)=z(t),
\end{aligned}
$$




$$
\begin{aligned}
z^{\prime}(t)= & a(t) \varphi(z(t-\tau)) z(t)+b(t) \psi(y(t)) \\
& +h(x(t))+f(x(t), y(t-\tau))+p(t) .
\end{aligned}
$$

Let $\mathrm{g}(\mathrm{x}(\mathrm{t}), \mathrm{y}(\mathrm{t}-\tau))=h(x(t))+f(x(t), y(t-\tau))$.

Hence, we have

$$
\begin{aligned}
x^{\prime}(t) & =y(t), \\
y^{\prime}(t) & =z(t), \\
z^{\prime}(t) & =a(t) \varphi(z(t-\tau)) z(t)+b(t) \psi(y(t))+g(x(t), y(t-\tau))+p(t),
\end{aligned}
$$

which implies that

$$
\begin{aligned}
x^{\prime}(t)= & y(t) \\
y^{\prime}(t)= & z(t), \\
z^{\prime}(t)= & a(t) \varphi(z(t-\tau)) z(t)+b(t) \psi(y(t))+g(x(t), y(t)) \\
& -\int_{-\tau}^{0} g_{y}(x(t), y(t+\sigma)) z(t+\sigma) d \sigma+p(t),
\end{aligned}
$$

where

$$
g_{y}=\frac{\partial g}{\partial y}
$$

Assume that:

(C1) $a_{0} \geq a(t) \geq 1, b_{0} \geq b(t) \geq 1, b^{\prime}(t) \geq 0$, where $a_{0}, b_{0} \in \Re$;

$(\mathrm{C} 2) v(t+\tau)\left[f(x(t), 0)+h^{\prime}(x(t)) x(t)\right] \leq 0$;

(C3) $\int_{0}^{v(t+\tau)}[f(x(t), u) d u-f(x(t), 0)] v(t+\tau)<0$ for $v(t+\tau) \neq 0$;

(C4) $v(t+\tau)\left[h^{\prime}(x) v(t+\tau)+\int_{0}^{v(t+\tau)} f_{x}(x(t), u) d u\right] \geq 0$;

(C5) $\left|f_{v}(x, v)\right|<P<\infty$;

(C6) $a+\tau \alpha<0, a, \tau, \alpha \in \Re, a<0, \tau>0, \alpha>0$;

(C7) $4 \alpha(a+\tau \alpha)+\tau P^{2}<0$.

Define the function $H(x, y)$ by

$$
H(x, y)=-\int_{0}^{y} g(x, u) d u-\frac{1}{2} b y^{2}, b \in \Re, b<0,(x, y) \in \Omega_{0}
$$




$$
\Omega_{0}=\{(x(t), y(t)):(x(t), y(t+\tau)) \in \Omega, \quad t \geq 0\},
$$

and $\Omega_{0}$ is a domain of the two dimensional Euclidean space $\Re^{2}$.

Lemma 2.1. Assume that

(D1) $\psi(0)=0, \frac{\psi(y)}{y} \leq b$ for $y \neq 0$, where $b \in \Re, b<0$;

(D2) $y g(x, 0) \leq 0$ for $x, y$, and $\int_{0}^{y} g(x, u) d u-g(x, 0) y<0$ for $x, y \neq 0$.

Then, the function $H(x, y)=L x^{2}+2 M x y+N y^{2}$ is positive definite and decrescent, where

$$
\begin{gathered}
L=L(x, y)=\frac{1}{x^{2}}\left[-\int_{0}^{y} g(x, u) d u+\int_{0}^{y} g(x, 0) d u\right], \\
M=M(x)=-\frac{1}{2 x} g(x, 0), \text { and } N=-\frac{1}{2} b .
\end{gathered}
$$

Proof. By noting the assumptions of Lemma 2.1, it follows that

$$
\begin{gathered}
L=\frac{1}{x^{2}}\left[-\int_{0}^{y} g(x, u) d u+\int_{0}^{y} g(x, 0) d u\right]>0, \\
2 M x y=-y g(x, 0) \geq 0
\end{gathered}
$$

and

$$
N y^{2}=-\frac{1}{2} b y^{2} \geq 0
$$

Then, we can conclude that

$$
H(x, y) \geq K\left(x^{2}+y^{2}\right),
$$

where $K=\min \left\{[\inf L(x, y)]\right.$ for all $\left.x, y \in \Omega_{0}, N\right\}, K>0$. This means that $H(x, y)$ is positive definite. It is also clear that the quadratic form $H(x, y)$ can be rearranged as

$$
H(x, y)=[x, y] T(x, y)\left[\begin{array}{l}
x \\
y
\end{array}\right]
$$

where 


$$
\mathrm{T}(\mathrm{x}, \mathrm{y})=\left[\begin{array}{cc}
\frac{1}{x^{2}}\left[-\int_{0}^{y} g(x, u) d u+\int_{0}^{y} g(x, 0) d u\right] & -\frac{1}{2 x} g(x, 0) \\
-\frac{1}{2 x} g(x, 0) & -\frac{1}{2} b
\end{array}\right] .
$$

Let $\lambda_{1}(x, y)$ and $\lambda_{2}(x, y)$ denote the characteristic roots of the matrix $T(x, y)$. Then, it is clear that

$$
H(x, y) \leq K^{\frac{1}{2}}\left(x^{2}+y^{2}\right),
$$

where $K=\sup \left[\lambda_{1}^{2}(x, y)+\lambda_{2}^{2}(x, y)\right]$ for all $x, y \in \Omega_{0}$, and $K>0$. Thus, the function $H(x, y)=L x^{2}+2 M x y+N y^{2}$ is decrescent. This completes the proof of Lemma 2.1.

Theorem 2.1. Assume that $p(t) \equiv 0$, conditions $(C 1)-(C 7)$ hold, and $\psi(0)=0, \frac{\psi(y)}{y} \leq b$ for $y \neq 0, b \in \Re, b<0 ; \varphi(z(t-\tau)) \leq a$ for $z(t-\tau), a \in$ $\Re, a<0$.

Then, the zero solution of equation (2.1) is stable.

Proof. We define the Lyapunov-Krasovskii functional [13] $V=V\left(t, x_{t}, y_{t}, z_{t}\right)$ by

$(2.3) V=-\int_{0}^{y} g(x, u) d u-b(t) \int_{0}^{y} \psi(u) d u+\frac{1}{2} z^{2}+\alpha \int_{-\tau}^{0}\left[\int_{\theta}^{0} z^{2}(\sigma) d \sigma\right] d \theta$,

where

$\mathrm{g}(\mathrm{x}, \mathrm{y}(\mathrm{t}-\tau))=f(x, y(t-\tau))+h(x),(\mathrm{x}, \mathrm{y}) \in \Omega_{0}, z=\{z(t): z(t)=$ $\left.y^{\prime}(t), \quad t \geq 0\right\}$, and $\alpha$ is a certain positive constant.

Consider the terms

$-\int_{0}^{y} g(x, u) d u-b(t) \int_{0}^{y} \psi(u) d u$, which are involved in (2.3).

It is clear that

$-\int_{0}^{y} g(x, u) d u-b(t) \int_{0}^{y} \psi(u) d u=-\int_{0}^{y} g(x, u) d u-b(t) \int_{0}^{y} \frac{\psi(u)}{u} u d u$ 


$$
\begin{aligned}
& \geq-\int_{0}^{y} g(x, u) d u-\int_{0}^{y} b u d u \\
& =-\int_{0}^{y} g(x, u) d u-\frac{1}{2} b y^{2}
\end{aligned}
$$

by the assumptions of Theorem 2.1 .

Then, it is clear that the Lyapunov-Krasovskii functional $V=V\left(t, x_{t}, y_{t}, z_{t}\right)$ is positive definite, and

$$
\begin{gathered}
V \geq-\int_{0}^{y} g(x, u) d u-\frac{1}{2} b y^{2}+\frac{1}{2} z^{2} \\
+\alpha \int_{-\tau}^{0}\left[\int_{\theta}^{0} z^{2}(\sigma) d \sigma\right] d \theta .
\end{gathered}
$$

In view of the above discussion and the functional $V$, we can conclude that

$$
K\left(x^{2}+y^{2}\right)+\frac{1}{2} z^{2}+\alpha \int_{-\tau}^{0}\left[\int_{\theta}^{0} z^{2}(\sigma) d \sigma\right] d \theta \leq V .
$$

Differentiating the functional $V$ with respect to $t$, we have

$$
\begin{aligned}
V^{\prime}= & -y \int_{0}^{y} g_{x}(x, u) d u+a(t) \varphi(z(t-\tau)) z^{2}-b^{\prime}(t) \int_{0}^{y} \psi(u) d u \\
& -\int_{-\tau}^{0} g_{y}(x(t), y(t+\sigma)) z(t) z(t+\sigma) d \sigma+\alpha \int_{-\tau}^{0}\left[z^{2}(t)-z^{2}(t+\sigma)\right] d \sigma .
\end{aligned}
$$

By the assumptions of Theorem 2.1, we have

$$
\begin{aligned}
V^{\prime} \leq & -y \int_{0}^{y} g_{x}(x, u) d u+\int_{-\tau}^{0}\left[\left(\frac{a}{\tau}+\alpha\right) z^{2}(t)-\alpha z^{2}(t+\sigma)\right] d \sigma \\
& -\int_{-\tau}^{0} g_{y}(x(t), y(t+\sigma)) z(t) z(t+\sigma) d \sigma
\end{aligned}
$$

where 


$$
\mathrm{g}_{x}=\frac{\partial g}{\partial x}, g_{y}=\frac{\partial g}{\partial y} \text {. }
$$

In view of the assumption $(\mathrm{C} 4)$, it follows that $y \int_{0}^{y} g_{x}(x, u) d u \geq 0$. Consider the terms $\int_{-\tau}^{0}\left[\left(\frac{a}{\tau}+\alpha\right) z^{2}(t)-\alpha z^{2}(t+\sigma)\right] d \sigma-\int_{-\tau}^{0} g_{y}(x(t), y(t+$ $\sigma)) z(t) z(t+\sigma) d \sigma$.

By noting assumptions (C4)-(C6), it can be seen that

$$
\begin{aligned}
-\alpha^{2}-\frac{a \alpha}{\tau}-\frac{1}{4} g_{y}^{2}(x(t), y(t+\sigma)) & =-\frac{4 \tau \alpha^{2}+4 a \alpha+\tau g_{y}^{2}(x(t), y(t+\sigma))}{\tau} \\
& \geq-\frac{4 \tau \alpha^{2}+4 a \alpha+\tau P^{2}}{\tau} .
\end{aligned}
$$

Therefore, if $4 \tau \alpha^{2}+4 a \alpha+\tau P^{2}<0$, then the quadratic form

$$
\begin{array}{r}
\alpha z^{2}(t+\sigma)+g_{y}(x(t), y(t+\sigma)) z(t+\sigma) z(t)-\left(\frac{a}{\tau}+\alpha\right) z^{2}(t) \\
=[z(t+\sigma), z(t)]\left[\begin{array}{cc}
\alpha & \frac{1}{2} g_{y}(x(t), y(t+\sigma)) \\
\frac{1}{2} g_{y}(x(t), y(t+\sigma)) & -\left(\frac{a}{\tau}+\alpha\right)
\end{array}\right]\left[\begin{array}{c}
z(t+\sigma) \\
z(t)
\end{array}\right]
\end{array}
$$

is positive for any $z(t+\sigma)$ and $z(t)$.

Then, we have $\mathrm{V}^{\prime} \leq 0$.

Thus, in view of the discussion made and Theorem 1.1, we can conclude that the zero solution of equation (2.1) is stable.

Remark 2.1. 2.1 If the assumptions of Theorem 2.1 hold, then

$$
\begin{aligned}
& K\left(x^{2}+y^{2}\right)+\frac{1}{2} z^{2}+\alpha \int_{-\tau}^{0}\left[\int_{\theta}^{0} z^{2}(\sigma) d \sigma\right] d \theta \leq V \\
\leq & K^{\frac{1}{2}}\left(x^{2}+y^{2}\right)+\frac{1}{2} z^{2}+\alpha \int_{-\tau}^{0}\left[\int_{\theta}^{0} z^{2}(\sigma) d \sigma\right] d \theta .
\end{aligned}
$$

Hence, we can conclude that the zero solution of equation (2.1) is uniformly stable.

Finally, for the case $p(t) \neq 0$, we prove the following theorem.

Theorem 2.2. In addition to conditions $(C 1)-(C 7)$, assume that $p \in$ $L^{1}(0, \infty)$. Then, all solutions of equation (2.1) are bounded. 
Proof. For the case $p(t) \neq 0$, it is easy to see from $V$, which is given in (2.3), that

$$
V^{\prime} \leq z p(t)
$$

Then, we have

$$
V^{\prime} \leq|z||p(t)| \leq\left(1+z^{2}\right)|p(t)|
$$

From the discussion made for (2.3), it follows that $\mathrm{K}\left(\mathrm{x}^{2}+y^{2}\right)+\frac{1}{2} z^{2} \leq V$.

Hence, $\mathrm{V}^{\prime} \leq(1+2 V)|p(t)|$, and an application of Gronwall's inequality [4] bounds $V$.

Thus, all solutions of (2.1) are bounded.

Remark 2.2. If the assumptions of Theorem 2.1 hold, then

$$
\begin{aligned}
& K\left(x^{2}+y^{2}\right)+\frac{1}{2} z^{2}+\alpha \int_{-\tau}^{0}\left[\int_{\theta}^{0} z^{2}(\sigma) d \sigma\right] d \theta \leq V \\
\leq & K^{\frac{1}{2}}\left(x^{2}+y^{2}\right)+\frac{1}{2} z^{2}+\alpha \int_{-\tau}^{0}\left[\int_{\theta}^{0} z^{2}(\sigma) d \sigma\right] d \theta .
\end{aligned}
$$

Hence, we can conclude that all solutions of equation (2.1) are uniformly bounded.

\section{Conclusion}

A kind of functional differential equations of third order with retarded argument has been considered. Defining an appropriate Lyapunov-Krasovskii functional [13], stability, uniformly stability, boundedness and uniformly boundedness of solutions have been investigated. Our results complement and improve some recent ones.

\section{References}

[1] Adams, D. O.; Omeike, M. O.; Mewomo, O. T.; Olusola, I. O., Boundedness of solutions of some third order non-autonomous ordinary differential equations. J. Nigerian Math. Soc. 32, pp. 229-240, (2013). 
[2] Ademola, T. A.; Arawomo,P. O., Asymptotic behaviour of solutions of third order nonlinear differential equations. Acta Univ. Sapientiae Math. 3, No. 2, pp. 197-211, (2011).

[3] Afuwape, A. U.; Adesina, O. A., On the bounds for mean-values of solutions to certain third-order non-linear differential equations. Fasc. Math. No. 36, pp. 5-14, (2005).

[4] Ahmad, S.; Rama Mohana Rao, M., Theory of ordinary differential equations. With applications in biology and engineering. Affiliated East-West Press Pvt. Ltd., New Delhi, (1999).

[5] Bai, Yuzhen; Guo, C., New results on stability and boundedness of third order nonlinear delay differential equations. Dynam.Systems Appl. 22, No. 1, pp. 95-104, (2013).

[6] Burton, T. A., Stability and Periodic Solutions of Ordinary and Functional Differential Equations. Academic Press, Orlando, (1985).

[7] Chlouverakis, K. E.; Sprott, J. C., Chaotic hyperjerk systems. Chaos Solitons Fractals 28, No. 3, pp. 739-746, (2006).

[8] Cronin-Scanlon, J., Some mathematics of biological oscillations. SIAM Rev. 19, No. 1, pp. 100-138, (1977).

[9] Eichhorn, R.; Linz, S. J.; Hänggi, P., Transformations of nonlinear dynamical systems to jerky motion and its application to minimal chaotic flows. Phys Rev E58, pp. 7151-7164, (1998).

[10] Elhadj, Z.; Sprott, J. C., Boundedness of certain forms of jerky dynamics. Qual. Theory Dyn.Syst. 11, No. 2, pp. 199-213, (2012).

[11] El-Nahhas, A., Stability of a third-order differential equation of retarded type. Appl. Math. Comput. 60, No. 2-3, pp. 147-152, (1994).

[12] Èl'sgol'ts, L. È., Introduction to the theory of differential equations with deviating arguments. Translated from the Russian by Robert J. McLaughlin Holden-Day, Inc., San Francisco, Calif.-LondonAmsterdam, (1966).

[13] Krasovskii, N. N., Stability of motion. Applications of Liapunov's second method to differential systems and equations with delay. Stanford, Calif.: Stanford University Press, (1963). 
[14] Linz, S. J., Onhyperjerky systems. Chaos Solitons Fractals 37, No. 3, pp. 741-747, (2008).

[15] Ogundare, B. S.; Okecha, G.E., On the boundedness and the stability of solution to third order non-linear differential equations. Ann. Differential Equations 24, No. 1, 1-8, (2008).

[16] Rauch, L. L., Oscillation of a third order nonlinear autonomous system. Contributions to the Theory of Nonlinear Oscillations, pp. 39-88. Annals of Mathematics Studies, No. 20. Princeton University Press, Princeton, N. J., (1950).

[17] Reissig, R.; Sansone, G.; Conti, R., Non-linear differential equations of higher order. Translated from the German. Noordhoff International Publishing, Leyden, (1974).

[18] Sadek, A. I., On the stability of solutions of some non-autonomous delay differential equations of the third order. Asymptot.Anal. 43,no. 1-2, pp. 1-7, (2005).

[19] Smith, H., An introduction to delay differential equations with applications to the life sciences. Texts in Applied Mathematics, 57. Springer, New York, (2011).

[20] Tunç, C., Uniform ultimate boundedness of the solutions of third-order nonlinear differential equations. Kuwait J. Sci. Engrg. 32, No. 1, 39-48, (2005).

[21] Tunç, C., On the stability of solutions to a certain fourth-order delay differential equation. Nonlinear Dynam. 51(2008), No. 1-2, pp. 71-81, (2008).

[22] Tunç, C., On the stability and boundedness of solutions to third order nonlinear differential equations with retarded argument.Nonlinear Dynam. 57, No. 1-2, pp. 97-106, (2009).

[23] Tunç, C., Bound of solutions to third-order nonlinear differential equations with bounded delay. J. Franklin Inst. 347, No. 2, pp. 415-425, (2010).

[24] Tunç, C., On the stability and boundedness of solutions of nonlinear third order differential equations with delay. Filomat 24, No. 3, pp. 1-10, (2010). 
[25] Tunç, C., On the qualitative behaviors of solutions of some differential equations of higher order with multiple deviating arguments. J. Franklin Inst. 351, No. 2, 643-655, (2014).

[26] Tunç, C., Stability and boundedness of the nonlinear differential equations of third order with multiple deviating arguments. Afr. Mat. 24, No. 3, pp. 381-390, (2013).

[27] Tunç, C., Stability and boundedness for a kind of non-autonomous differential equations with constant delay. Appl. Math. Inf. Sci. 7, No. 1, pp. 355-361, (2013).

[28] Yoshizawa,T., Stability theory by Liapunov's second method. Publications of the Mathematical Society of Japan, No. 9. The Mathematical Society of Japan, Tokyo, (1966).

[29] Zhang, Lijuan; Yu, Lixin, Global asymptotic stability of certain thirdorder nonlinear differential equations. Math.Methods Appl. Sci. 36, No. 14, pp. 1845-1850, (2013).

\author{
Cemil Tunç \\ Department of Mathematics, \\ Faculty of Sciences, \\ Yüzüncü Yil University, \\ 65080, Van - Turkey \\ Turkey \\ e-mail : cemtunc@yahoo.com
}

